\title{
Premières investigations et expérimentations sur la tenue à l'érosion-corrosion des pompes alimentaires des générateurs de vapeur
}

\author{
First investigations and experiments on resistance \\ to erosion-corrosion in steam-generator feed pumps
}

\author{
L. Randrianarivo
}

Département Machines et Structures

Direction des Études et Recherches d'Électricité de France (Chatou)

\section{Introduction}

Ces dernières années, Electricité de France a rencontré un certain nombre de problèmes de dégradations par érosion-corrosion au niveau des corps des pompes alimentaires des tranches thermiques classiques de 250 et $600 \mathrm{MW}$ et plus récemment, des tranches nucléaires de la filière PWR $900 \mathrm{MW}$.

Ces dégradations peuvent avoir des conséquences graves car, après une période d'incubation, leur évolution dans le temps semble être très rapide. Il convient de noter que ces dégradations se sont traduites par des pertes d'épaisseur pouvant atteindre $15 \mathrm{~mm}$ après 30000 à 40000 heures de fonctionnement sur la quasitotalité des corps de pompes alimentaires des tranches classiques et $10 \mathrm{~mm}$ environ après 10000 heures pour les tranches nucléaires.

Ce type de matériel fonctionne généralement dans des conditions de températures comprises entre 160 et $180^{\circ} \mathrm{C}$ et de $\mathrm{pH}$ comprises entre 8,8 et 9,2 .

Devant le caractère pratiquement systématique de ces dégradations et du fait que les pompes alimentaires ont des caractéristiques de plus en plus poussées (facteur aggravant quant aux vitesses d'écoulement) EDF, en collaboration avec les constructeurs de pompes, a entrepris une analyse systématique des dégradations constatées et engagé une recherche expérimentale afin de dégager les paramètres influents sur ce phénomène.

Description succinte des pompes alimentaires

Les pompes alimentaires des tranches classiques aspirent l'eau dans une bâche alimentaire pour la refouler dans le générateur de vapeur au travers des réchauffeurs du poste d'eau HP (Fig. 1).

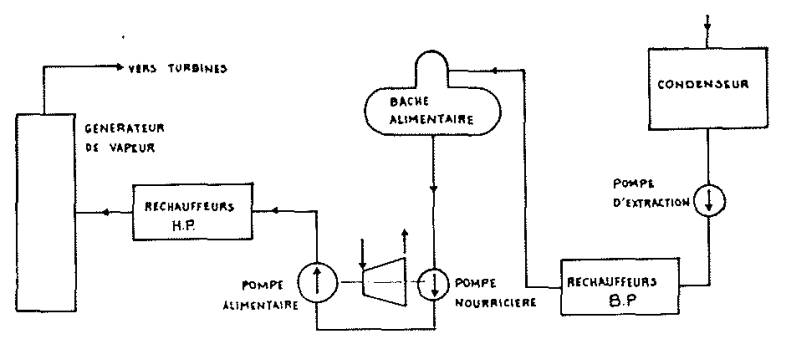

Figure 1 - Schéma de principe du poste d'eau des tranches thermiques classiques.

Elles sont constituées généralement par l'association de deux pompes en série, l'une nourricière et l'autre alimentaire.

La pompe nourricière est du type centrifuge à simple ou double volute avec une roue à simple ou double ouïe. Le corps est obturé d'un côté par un flasque.

La pompe alimentaire est du type centrifuge multicellulaire avec un corps cylindrique forgé.

Ces pompes ont un débit horaire de 800 tonnes pour les tranches $250 \mathrm{MW}$ et 2000 tonnes pour celles de $600 \mathrm{MW}$.

Dans les tranches nucléaires PWR $900 \mathrm{MW}$, il existe deux schémas du poste d'eau : L'un est celui du CP1 de technologie américaine, et l'autre celui du CP2 de technologie française. Dans le premier cas, l'eau condensée est reprise aux puits du condenseur par les pompes d'extraction qui gavent directement deux pompes alimentaires disposées en parallèle au travers des réchauffeurs du poste d'eau BP (Fig. 2).

Dans le deuxième cas, on retrouve à peu près la même configuration de poste d'eau que dans les tranches classiques (Fig. 3). 


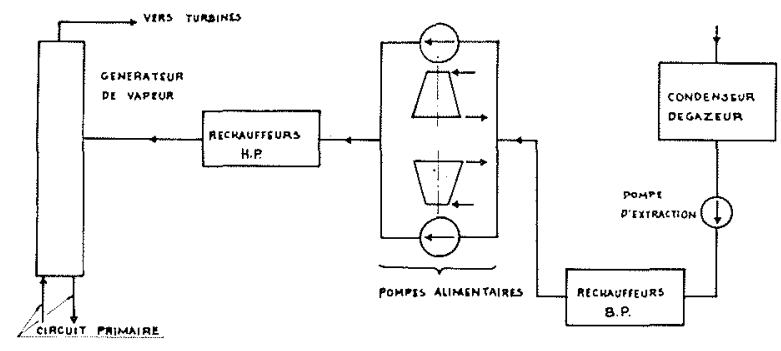

Figure 2 - Schéma de principe du poste d'eau du circuit secondaire des tranches nucléaires du CP1.

Les tranches du CP2 ne disposant pas encore d'un nombre d'heures de fonctionnement suffisamment significatif quant au phénomène d'érosion-corrosion, notre domaine d'observation couvre seulement, pour les tranches nucléaires, le parc des tranches $900 \mathrm{MW}$ du CP1.

Les pompes alimentaires de ces tranches sont du type monocellulaire (Fig. 4). Elles sont ainsi décrites :

- le stator est constitué par la volute à l'intérieur de laquelle vient se fixer un diffuseur rapporté (en acier inoxydable). Ce dernier se monte en prolongement du diffuseur fixe venu de fonderie avec la volute.

L'ouverture, côté opposé à l'accouplement, est obturée par un fond boulonné.

- le mobile est constitué de la roue à double entrée montée sur l'arbre.

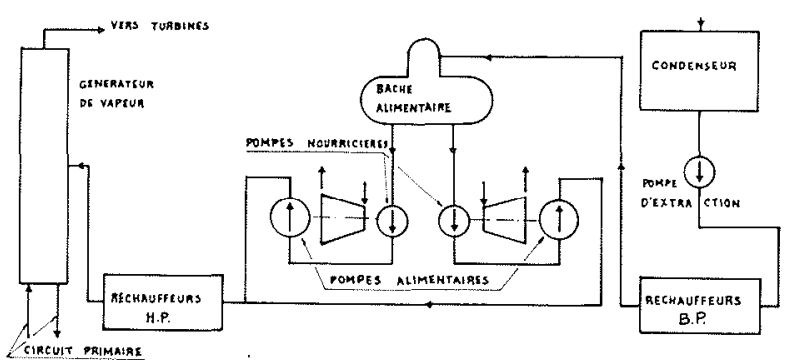

Figure 3 - Schéma de principe du poste d'eau du circuit secondaire des tranches nucléaires du CP2.

Ces pompes ont un débit horaire de 3004 tonnes au régime permanent maximal.

Constatations effectuées sur les pompes alimentaires des tranches thermiques classiques et nucléaires

L'érosion-corrosion dans les pompes alimentaires, se manifeste surtout au niveau du corps de pompe. Les dégradations constatées sont les suivantes:

\section{Tranches classiques}

\section{Pompes nourricières}

L'enveloppe des zones fréquemment érodées inclut la

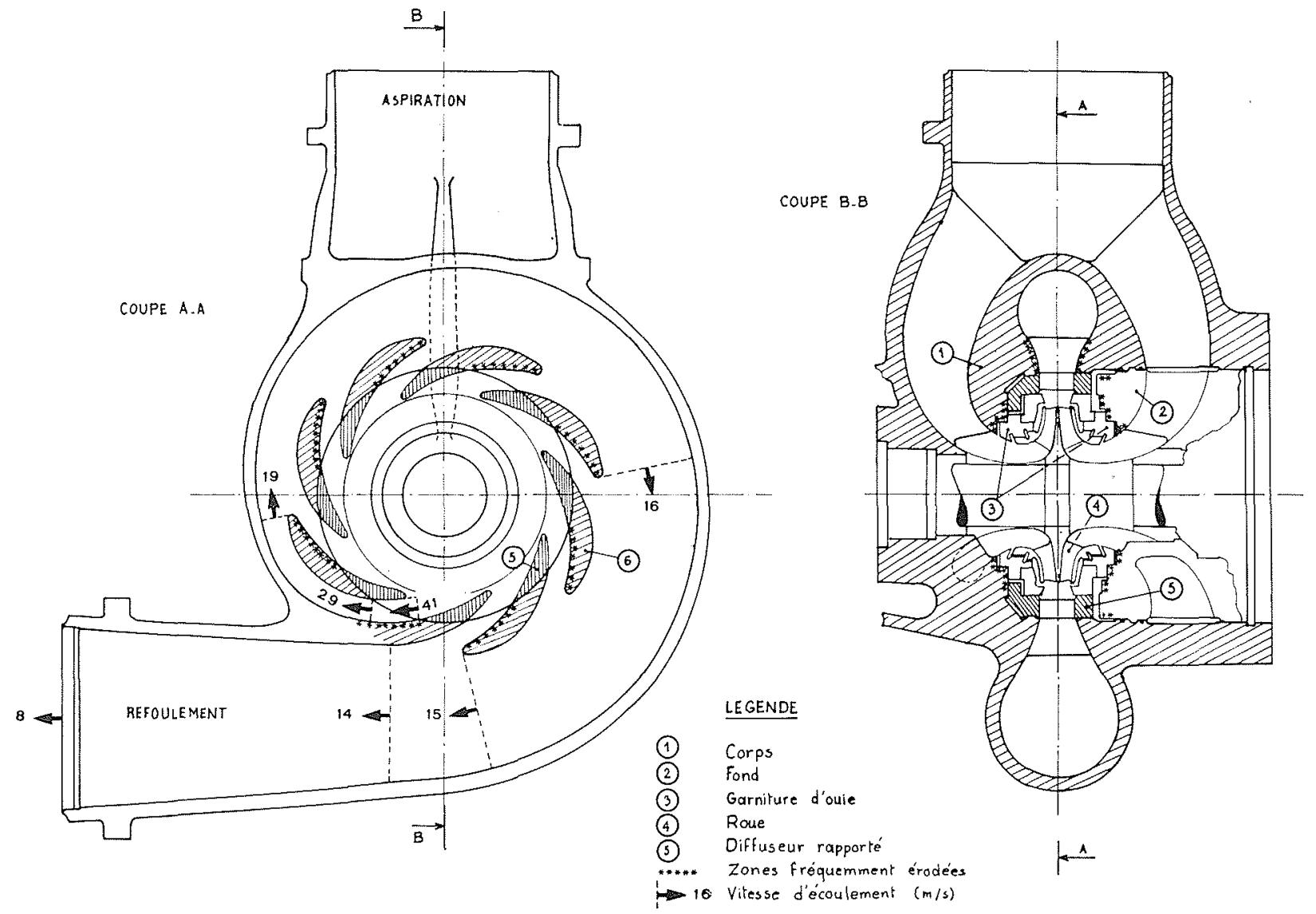

Figure 4 - Pompe alimentaire des tranches nucléaires. 
volute dans sa totalité (Fig. 5a) et le flasque du corps (Fig. 5b).

L'érosion-corrosion apparaît sous forme d'usures généralisées de 4 à $5 \mathrm{~mm}$ de profondeur avec à certains endroits notamment dans le voisinage du bec de volute des affouillements pouvant atteindre 10 à $15 \mathrm{~mm}$ de profondeur, voire $37 \mathrm{~mm}$ (Fig. 5).

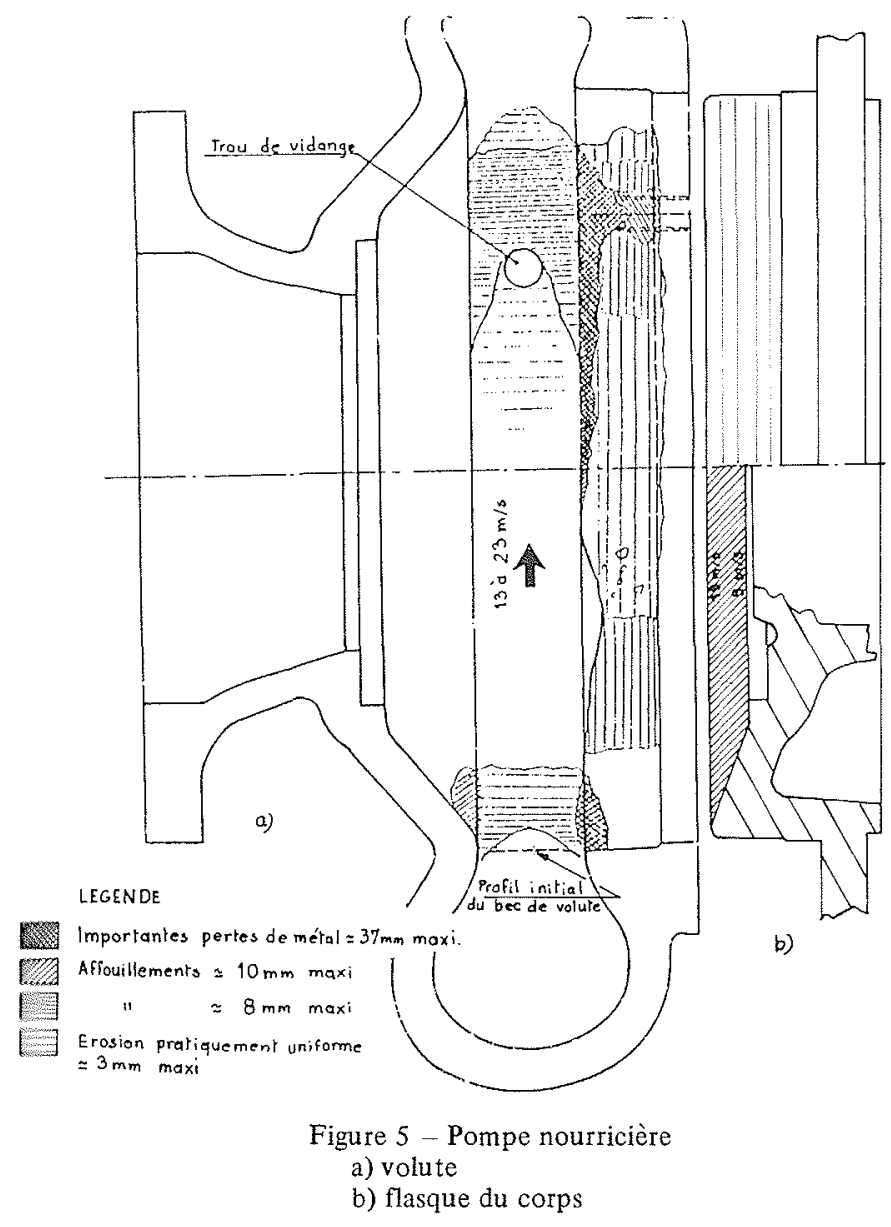

Les corps de ce type de pompe sont généralement réalisés en acier moulé du type A $48 \mathrm{CM}$.

L'ordre de grandeur des vitesses d'écoulement est pratiquement le même pour les tranches de $250 \mathrm{MW}$ et de $600 \mathrm{MW}$; il est de $23 \mathrm{~m} / \mathrm{s}$ dans le voisinage du bec de volute, de $13 \mathrm{~m} / \mathrm{s}$ à $23 \mathrm{~m} / \mathrm{s}$ dans la volute et de 9 à $19 \mathrm{~m} / \mathrm{s}$ dans le voisinage du flasque du corps.

\section{Pompes alimentaires}

L'enveloppe des zones fréquemment érodées inclut le voisinage du dernier diffuseur et l'entonnement du trou vers la tubulure de refoulement (Fig. 6).

Les dégradations se manifestent par une érosion assez importante au niveau de la tubulure de refoulement et de part et d'autre de celle-ci. La profondeur maximale des affouillements atteint $15 \mathrm{~mm}$ au voisinage immédiat d'un revêtement en acier inoxydable (ce revêtement est conçu dès l'origine sur ce type de pompe, il déborde légèrement les faces d'appui du cartouche).
Les corps de ce type de pompe sont généralement réalisés en acier forgé du type XC $18 \mathrm{f}$.

L'ordre de grandeur des vitesses d'écoulement est de $13 \mathrm{~m} / \mathrm{s}$ pour les tranches de $250 \mathrm{MW}$ et de $17 \mathrm{~m} / \mathrm{s}$ pour celles de $600 \mathrm{MW}$.

\section{Tranches Nucléaires}

Tout comme dans les tranches classiques, l'érosioncorrosion se manifeste surtout au niveau du corps de pompe. Ce dernier est actuellement réalisé en acier non allié moulé.

L'enveloppe des zones fréquemment érodées inclut la face d'appui de la garniture d'ouie, côté corps et côté fond, les intrados et les flancs des canaux des ailettes du diffuseur venu de fonderie avec la volute (Fig. 4).

Les dégradations se manifestent, dans les cas extrêmes, sous forme de cavités pouvant atteindre 20 à $30 \mathrm{~mm}$ au niveau de la face d'appui de la garniture d'ouie côté corps et sous forme d'érosion de 5 à $8 \mathrm{~mm}$ sur les intrados des ailettes et de $10 \mathrm{~mm}$ sur les flancs des canaux (Fig. 4).

L'ordre de grandeur des vitesses d'écoulement est de 19 à $40 \mathrm{~m} / \mathrm{s}$ dans les canaux du diffuseur, et de $70 \mathrm{~m} / \mathrm{s}$ dans le voisinage de la garniture d'ouie.

\section{Réparations}

\section{Tranches classiques}

La réparation des corps de pompes a consisté à effectuer un rechargement en acier inoxydable 24-12 des parties érodées.

Le principe est le suivant :

- affouillement jusqu'au métal sain des cavités en prenant soin d'obtenir des surfaces suffisantes afin de ne pas former des pastilles de métal d'appoint susceptibles de se détacher :

- ressuage des parties meulées;

- nettoyage des surfaces à recharger ;

- rechargement en deux couches avec électrodes à enrobage basique, en limitant la profondeur de pénétration de la première couche (risque de fissuration); - meulage des surfaces sans chercher à reconstituer le profil de base mais uniquement pour ne pas contrarier l'écoulement.

Le contrôle du dépôt se fait par ressuage de la première couche après blanchissage à la meule et de la deuxième couche après usinage.

\section{Tranches nucléaires}

Si le corps est réparable in situ, le procédé consiste à usiner (à l'aide d'une machine spéciale) et à recharger sous argon, avec le même type de métal que celui qui constitue le corps, les parties érodées. Dans le cas contraire, on remplace le corps défectueux. 


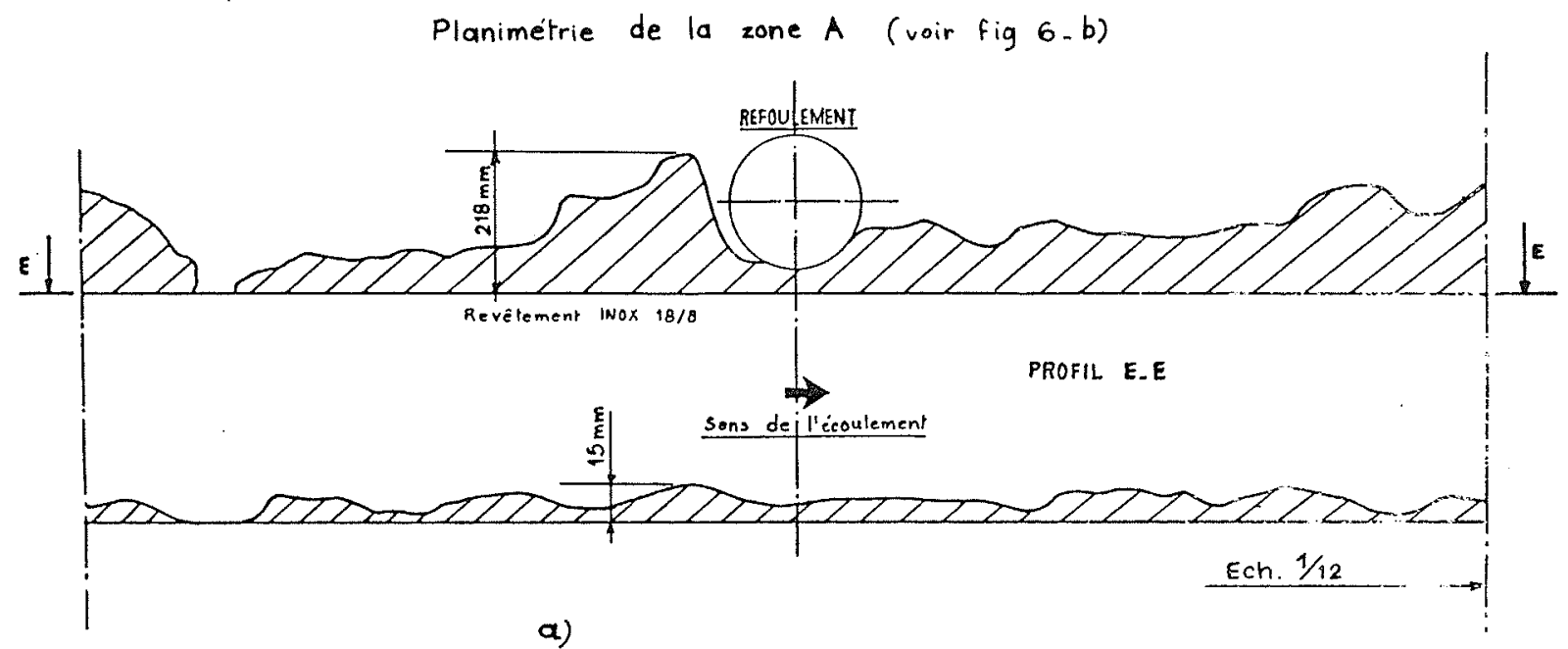

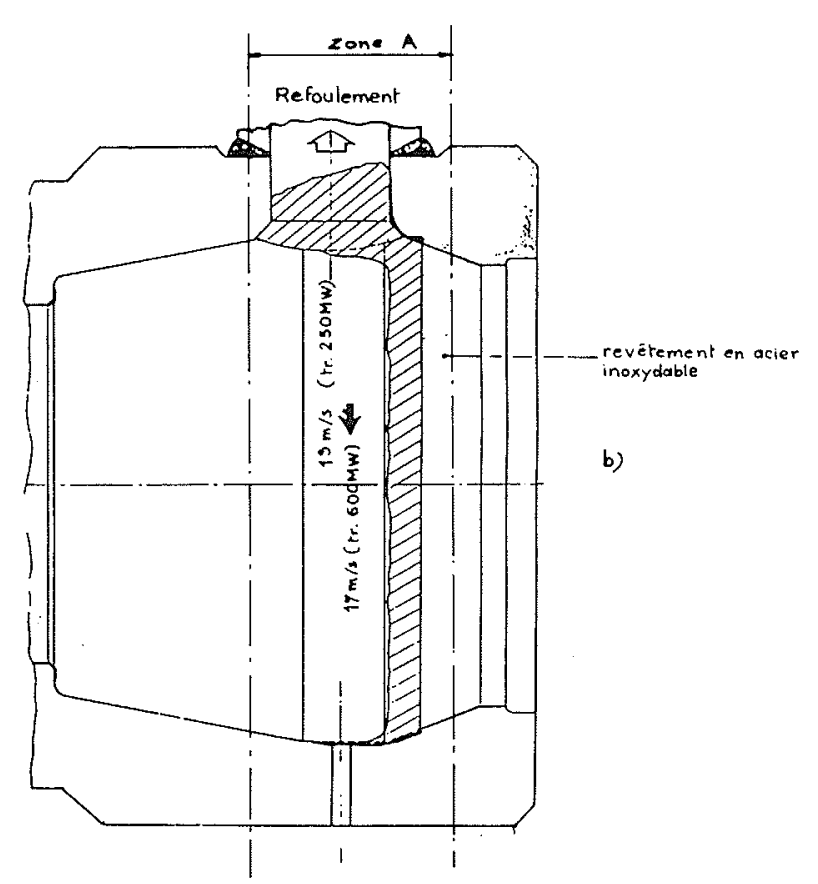

Figure 6 - Pompe alimentaire des tranches thermiques classiques a) planimétrie d'une zone érodée b) corps de pompe

\section{Politique actuelle d'EDF}

\section{Matériel en exploitation}

Visite systématique de ce type de matériel à partir de 30000 heures de fonctionnement pour les centrales thermiques classiques et à chaque rechargement (soit tous les ans) pour les tranches nucléaires (du moins pour le moment).

\section{Matériel en projet}

\section{Tranches classiques}

\section{Pompes nourricières}

Adoption d'un acier faiblement allié au chrome (te- neur en chrome comprise entre 0,40 et 2,60 ) voire même d'un acier fortement allié du type 13-1 ou 13-4.

\section{Pompes alimentaires}

Pour les corps en acier non allié, mise en place d'un revêtement en 18-8 ou 24-12 largement dimensionné (tubulure de refoulement comprise).

On envisage également l'utilisation du même type d'acier faiblement allié que pour les pompes nourricières.

\section{Tranches nucléaires}

Adoption d'un acier fortement allié au chrome (du type 13-1 ou 13-4).

\section{Recherches expérimentales}

Les études expérimentales que nous entreprenons ont pour but de rechercher les matériaux résistant le mieux aux dégradations imputables au phénomène d'érosion-corrosion dans les conditions de fonctionnement des matériels en centrale. Cette recherche est faite parmi les matériaux pouvant être envisagés pour la construction de ces matériels (corps de pompes par exemple).

D'autres études expérimentales qui ont pour but d'étudier l'influence des paramètres liés au fluide tels que vitesse, température, caractéristiques chimiques... sur le phénomène d'érosion-corrosion sont de même entreprises actuellement au sein d'EDF (DER-RNETC Les Renardières).

\section{Description sommaire de la boucle d'essais}

Cette boucle installée en dérivation sur le circuit alimentaire d'une tranche $125 \mathrm{MW}$ de la Centrale thermique de Creil (Fig. 7) doit permettre d'étudier l'influence de la vitesse du fluide sur le comportement des matëriaux à l'érosion-corrosion, les autres paramètres (température, pression, nature du fluide) sont fixés par les besoins de l'exploitation de la centrale. 


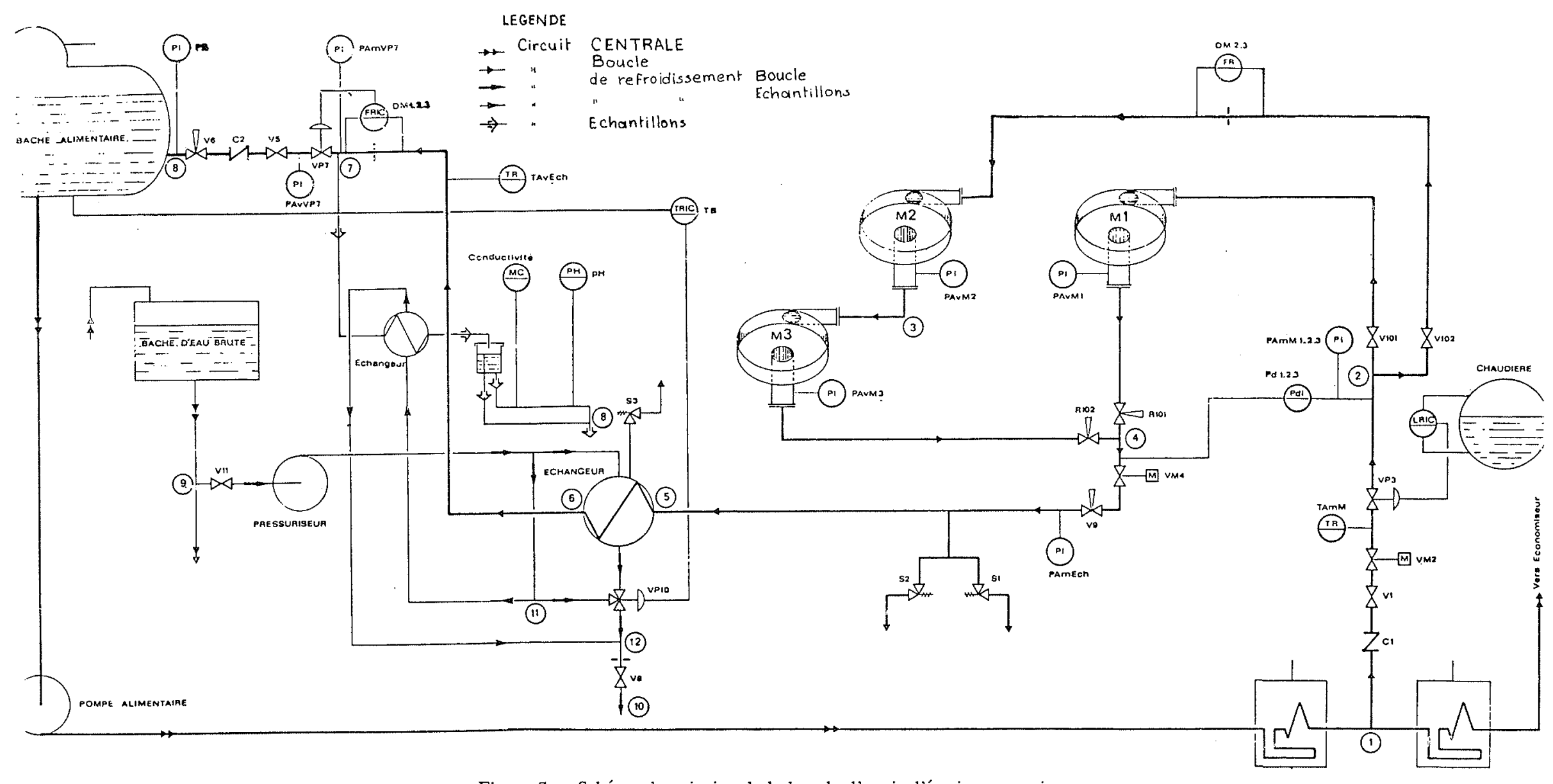

Figure 7 - Schéma de principe de la boucle d'essais d'érosion-corrosion. 

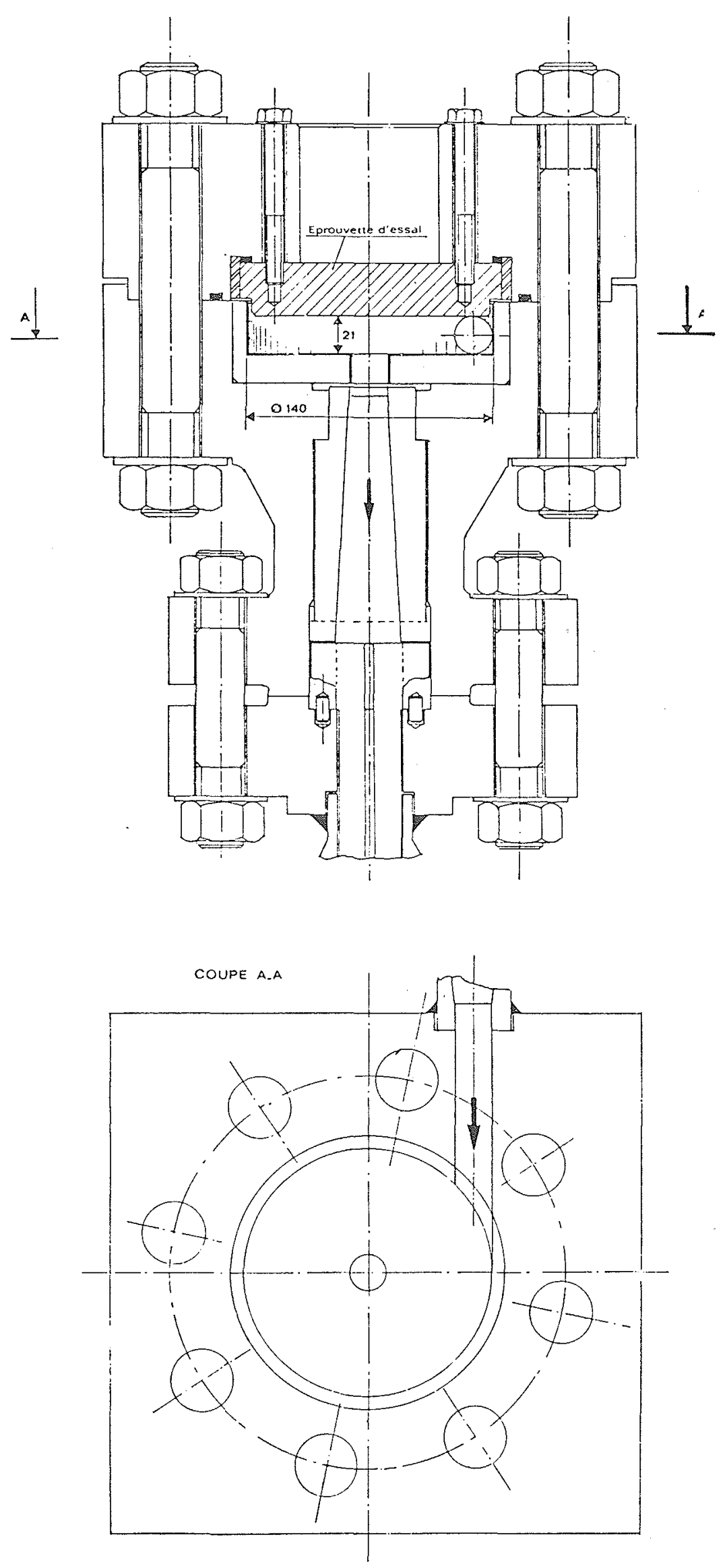

Figure 8-Maquette d'essais. 
L'eau alimentaire est prélevée à la sortie du premier réchauffeur HP situé en aval des pompes alimentaires, à une température de $205^{\circ} \mathrm{C}$ et à une pression de 118 bars.

Après passage dans la cellule d'essais, faisant également office de déchargeur, elle est réinjectée à la bâche alimentaire à $150^{\circ} \mathrm{C}$ et 5 bars environ après avoir traversé un échangeur.

La cellule d'essais est composée de trois veines. Chaque veine se compose d'un boitier cylindrique comportant une alimentation tangentielle et une évacuation axiale qui permettent de créer dans le boitier un écoulement du type vortex. (Fig. 8). Dans cet écoulement, la vitesse tangentielle du fluide croit de la périphérie vers le centre du boitier de façon continue (Fig. 9), ce qui doit permettre de déterminer la vitesse à partir de laquelle commence la dégradation par érosion-corrosion. Le fond du boitier, dont une face est soumise à l'action de l'écoulement, est démontable et constitue l'éprouvette d'essai. (Fig. 8).

Dans la configuration choisie, mise au point sur une maquette [1](1) et pour un débit d'alimentation de $20 \mathrm{t} / \mathrm{h}$, les vitesses s'échelonnent de $20 \mathrm{~m} / \mathrm{s}$ à $100 \mathrm{~m} / \mathrm{s}$.

\section{Premiers résultats d'essais}

Après la période de mise au point de la boucle d'essais, il a été effectué à ce jour 1560 heures d'essais dont 1260 heures consacrées à un acier non allié forgé du type XC48 et 310 heures à un acier moulé du type $\mathrm{A} 48 \mathrm{CM}$.

La figure 10 donne une indication sur l'évolution des pertes d'épaisseurs, évaluées à l'aide d'un comparateur, au bout de 100, 316 et 1260 heures pour l'acier XC48 et 310 heures pour l'acier A48CM (l'essai de ce

(1) Ce chiffre entre crochets se rapporte à la bibliographie annexée à cet article. matériau serait porté à 1260 heures comme pour l'acier $\mathrm{XC48}$ ). Nous en avons déduit une image des vitesses de corrosion en fonction du temps pour différentes vitesses d'écoulement (Fig. 11).

Notons que les zones exploitables (Fig. 9) de l'éprouvette sont repérées $\mathrm{A}$ et $\mathrm{B}$ et que les zones repérées $C$ et $D$ sont celles exposées au noyau de cavitation centrale (phénomène inhérent à un écoulement du type vortex).

L'examen de ces premiers résultats nous a permis de faire les constatations suivantes:

- Jusqu'aux environs de 300 heures d'essais, les pertes de métal sont très nettes quelle que soit la vitesse d'écoulement (l'anomalie constatée sur l'acier XC48 dans le voisinage de la zone $B$ côté zone $C$ (Fig. 9 et 10a, b) semble avoir comme origine un fonctionnement en régime diphasique lors de la première mise en service de la boucle d'essais, ce phénomène ne s'est pas produit pendant les essais de l'acier A48CM (Fig. 10c, d).

- De 300 à 1260 heures (Acier XC48), les pertes de métal étaient très faibles dans la zone $A$, où les vitesses d'écoulement varient à peu près de $20 \mathrm{~m} / \mathrm{s}$ à $40 \mathrm{~m} / \mathrm{s}$, et dans la zone B, elles sont relativement plus importantes, les vitesses d'écoulement variant à peu près de $40 \mathrm{~m} / \mathrm{s}$ à $77 \mathrm{~m} / \mathrm{s}$.

Ces constatations semblent confirmer la théorie selon laquelle le processus d'oxydation de l'acier en présence d'eau dégazée à haute température et à $\mathrm{pH}$ élevé peut aboutir à la formation d'une double couche de magnétite si la vitesse d'écoulement le permet.

Rappelons, très brièvement, que le fer réagit avec l'eau pour donner de l'hydroxyde ferreux qui lui-même se décompose pour donner de la magnétite $\mathrm{Fe} 304$ (réaction de Schikkor) qui constitue la première couche d'oxyde sur la surface du métal. Le dégagement d'hydrogène qui accompagne ces deux réactions peut entraîner une réduction des ions ferriques de la magnétite en ions
Figure 9 - Profils de vitesse. o vitesse minimale, $\nabla$ vitesse maximale.

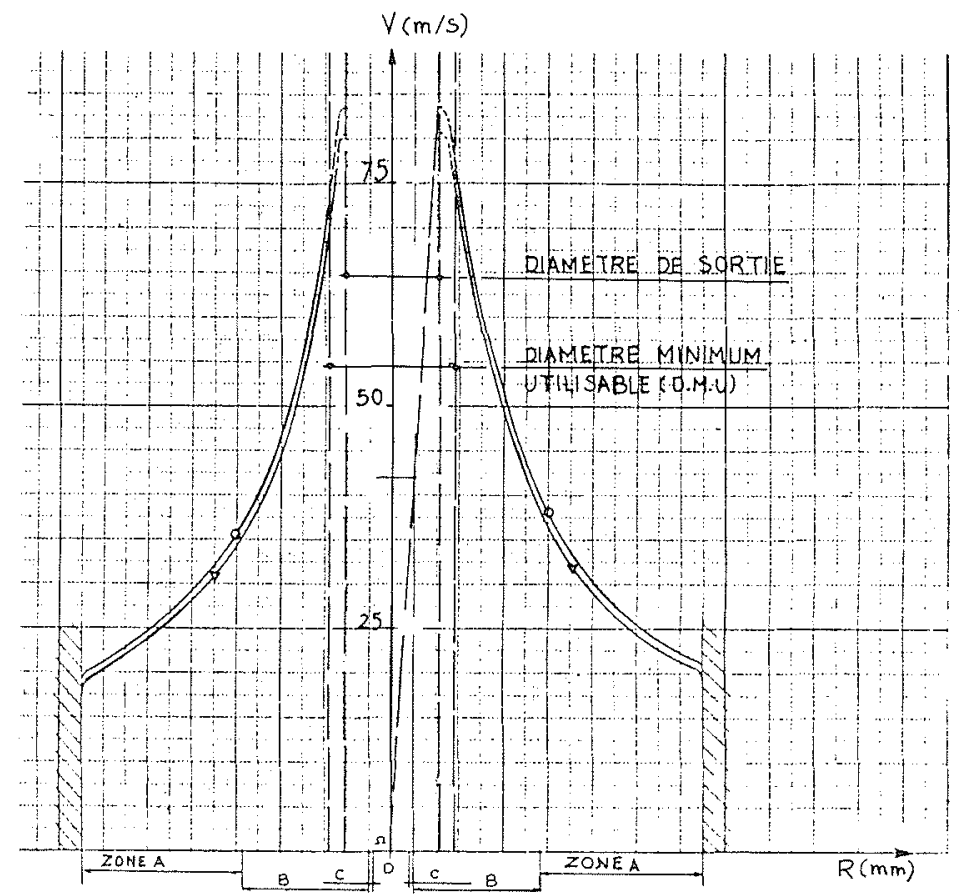



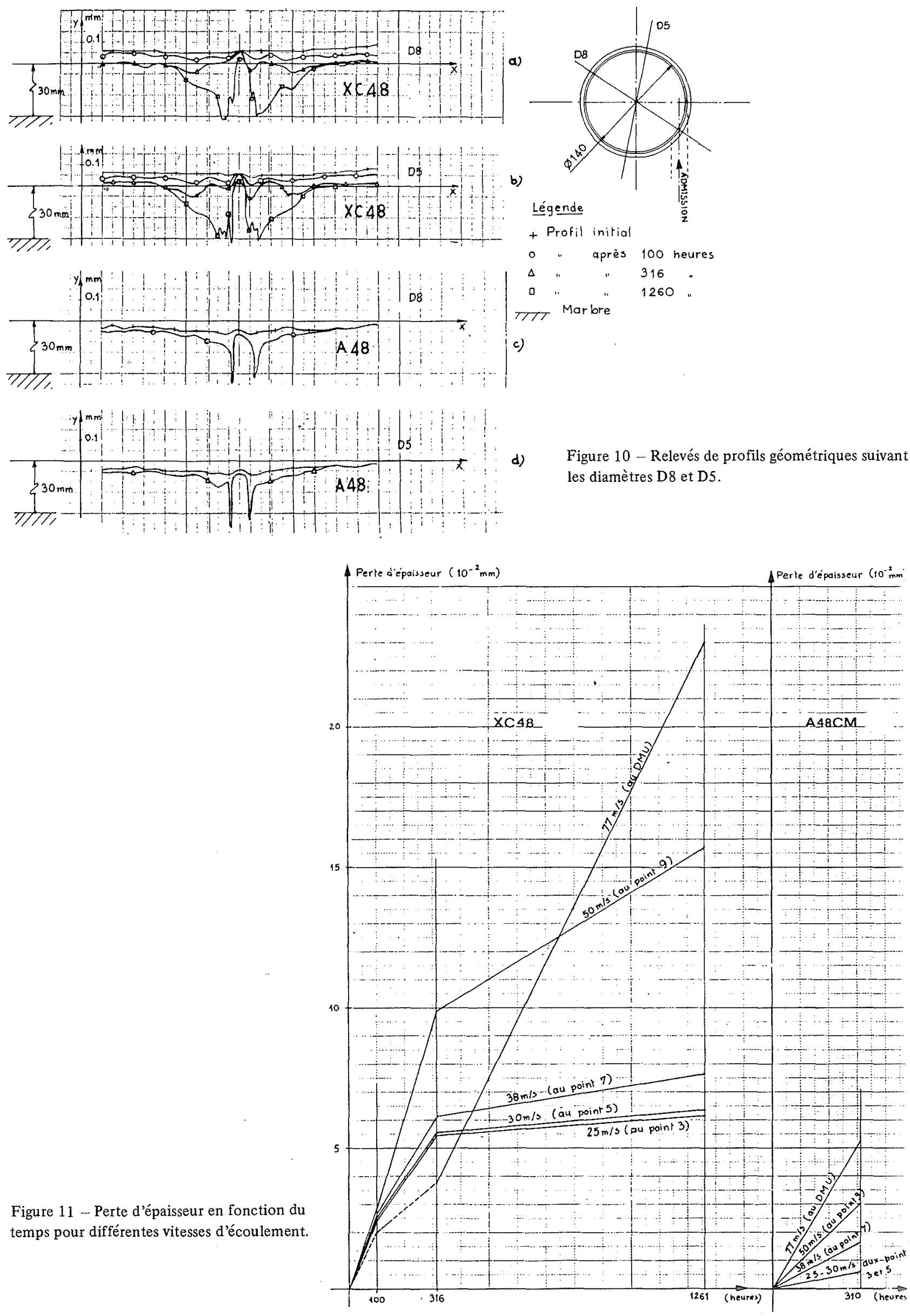
ferreux (réaction inverse de celle de Schikkor). Ces ions diffusent par différence de concentration vers la solution et précipitent sous forme de magnétite sur la première couche déjà formée, créant ainsi une couche externe d'oxyde pouvant assurer à la paroi métallique une certaine protection.

C'est ce phénomène qui semble s'être produit (entre 300 et 1260 heures) dans la zone $A$ où les pertes de métal sont très faibles. Par contre, dans la zone $B$ où elles étaient beaucoup plus importantes, les vitesses d'écoulement trop élevées n'ont pas permis aux ions ferreux de précipiter, empêchant ainsi la formation du film de passivation.

\section{Conclusion}

L'avènement des tranches nucléaires de la filière PWR $900 \mathrm{MW}$ a confirmé l'influence néfaste d'une augmenta- tion de la vitesse d'écoulement quant au comportement des corps de pompes alimentaires en acier non allié vis-à-vis du phénomène d'érosion-corrosion.

Les premières solutions envisagées par Electricité de France ont consisté, pour les matériels en projet, à adopter soit des aciers faiblement ou fortement alliés au chrome soit des aciers non alliés mais recouverts d'un revêtement en acier inoxydable.

Les études expérimentales en cours devront permettre de confirmer ou d'infirmer ces tendances.

\section{Référence bibliographique}

[1] BONAZZI A., LECOFFRE Y. - Définition d'une veine d'essai du type Vortex. Essais sur modèle transparent en eau froide. Neyrtec, Grenoble, Alsthom-Atlantique. Rapport d'études R. 20042, mars 1977.

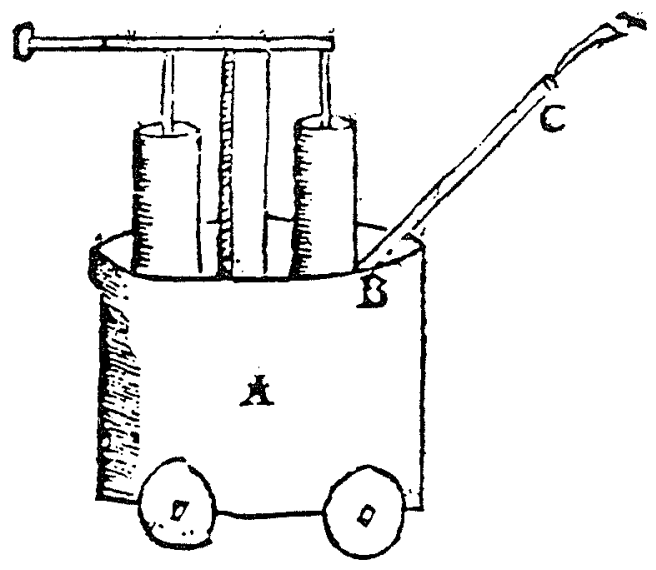




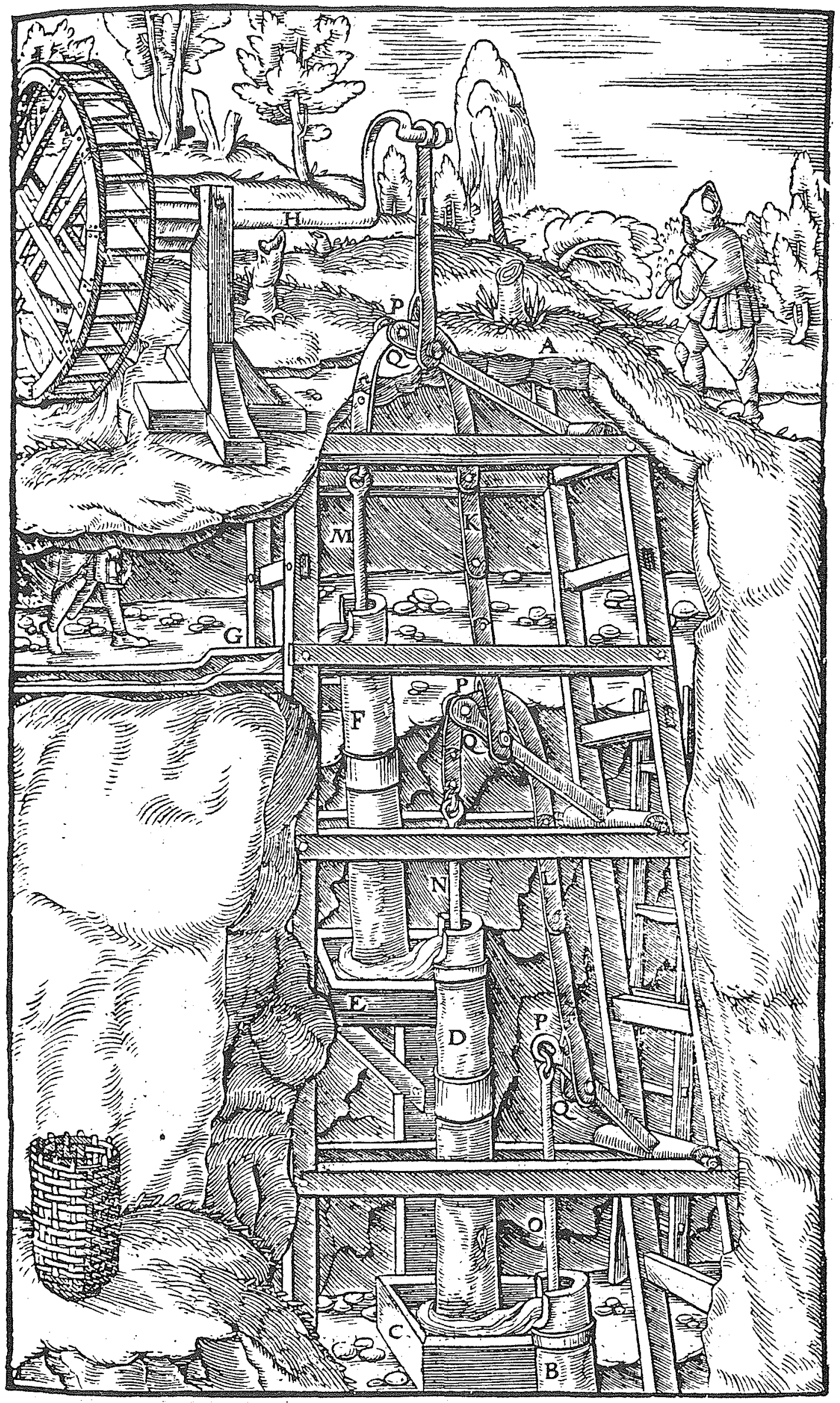

